\title{
C-peptide is internalised in human endothelial and vascular smooth muscle cells via early endosomes
}

\author{
P. Luppi $\cdot$ X. Geng $\cdot$ V. Cifarelli $\cdot$ P. Drain $\cdot$ M. Trucco
}

Received: 14 May 2009 /Accepted: 1 July 2009/Published online: 7 August 2009

(C) Springer-Verlag 2009

\begin{abstract}
Aims/hypothesis There is increasing evidence that Cpeptide exerts intracellular effects in a variety of cells and could be beneficial in patients with type 1 diabetes. Exactly how C-peptide achieves these effects, however, is unknown. Recent reports showed that C-peptide internalised in the cytoplasm of HEK-293 and Swiss $3 \mathrm{~T} 3$ cells, where it was not degraded for at least $1 \mathrm{~h}$ after uptake. In this study, we investigated the hypothesis that C-peptide is internalised via an endocytic pathway and traffics to classic endocytic organelles, such as endosomes and lysosomes.

Methods We studied the internalisation of C-peptide in vascular endothelial and smooth muscle cells, two relevant targets of Cpeptide activity, by using Alexa Fluor-labelled C-peptide probes in living cells and immunohistochemistry employing confocal laser-scanning microscopy. To examine trafficking to subcellular compartments, we used fluorescent constructs tagged to RAB5A, member RAS oncogene family (RAB5A) to identify early endosomes, or to lysosomal-associated membrane protein 1 (LAMP1) to identify lysosomes.
\end{abstract}

Electronic supplementary material The online version of this article (doi:10.1007/s00125-009-1476-7) contains supplementary material, which is available to authorized users.

P. Luppi $(\bowtie) \cdot$ V. Cifarelli $\cdot$ M. Trucco

Division of Immunogenetics, Department of Pediatrics,

Children's Hospital of Pittsburgh of UPMC,

Rangos Research Center,

530 45th Street,

Pittsburgh, PA 15201, USA

e-mail: luppip@pitt.edu

X. Geng $\cdot$ P. Drain

Department of Cell Biology and Physiology,

University of Pittsburgh, School of Medicine,

Pittsburgh, PA, USA
Results C-peptide internalised in the cytoplasm of cells within punctate structures identified as early endosomes. Internalisation was clearly detectable after $10 \mathrm{~min}$ of incubation and was blocked at $4{ }^{\circ} \mathrm{C}$ as well as with excess of unlabelled C-peptide. A minor fraction of vesicles, which increased with culture time, co-localised with lysosomes. Uptake of C-peptide was reduced by monodansylcadaverine, a pharmacological compound that blocks clathrin-mediated endocytosis, and by nocodazole, which disrupts microtubule assembly.

Conclusions/interpretation C-peptide internalises in the cytoplasm of cells by endocytosis, as demonstrated by its localisation in early endosomes. Endosomes might represent a signalling station, through which C-peptide might achieve its cellular effects.

Keywords C-peptide · Endocytosis · Endosomes . Endothelial cells $\cdot$ Lysosomes $\cdot$ Smooth muscle cells

$\begin{array}{ll}\text { Abbreviations } \\ \text { EEA1 } & \text { Early endosome antigen 1 } \\ \text { HAEC } & \text { Human aortic endothelial cells } \\ \text { LAMP1 } & \text { Lysosomal-associated membrane protein 1 } \\ \text { MDC } & \text { Monodansylcadaverine } \\ \text { NF-kB } & \text { Nuclear factor kappaB } \\ \text { RAB5A } & \text { RAB5A, member RAS oncogene family } \\ \text { UASMC } & \text { Umbilical artery smooth muscle cells }\end{array}$

\section{Introduction}

Human C-peptide is a peptide of 31 amino acids that is released by pancreatic beta cells in equimolar amounts with insulin in response to elevated blood glucose levels 
(hyperglycaemia). Once secreted into the bloodstream, Cpeptide circulates at low nanomolar concentrations in healthy individuals, but it is absent in most patients with type 1 diabetes [1]. In recent years, C-peptide has been shown to exert insulin-independent biological effects on a variety of cells, where it affects the activation of several intracellular pathways, such as, but not limited to, those involved in cellular proliferation and inflammation [2-4]. Importantly, C-peptide has been demonstrated to be beneficial when administered as replacement therapy to type 1 diabetes patients who suffer from some diabetic complications [5-8]. How exactly C-peptide achieves its intracellular effects in target cells, however, is unknown.

Several years ago, C-peptide was shown to specifically bind to plasma membranes from rat pancreatic beta cells [9], human renal tubular cells [10], human fibroblasts and endothelial cells [11]. More recently, C-peptide was shown to bind and cross plasma membranes, localizing in the cytoplasm of HEK-293 cells and Swiss 3T3 fibroblasts [12], where it was detected up to $1 \mathrm{~h}$ after its uptake. Nuclear localisation of C-peptide in HEK-293 cells and Swiss $3 \mathrm{~T} 3$ fibroblasts has also been demonstrated by the same group [12]. These findings demonstrate that, once internalised in the cytoplasm, C-peptide is not rapidly degraded but remains intact, possibly interacting with subcellular components through which it might achieve its cellular effects.

The process of internalisation from the cell surface and the subcellular localisation of C-peptide in target cells have not yet been investigated. In particular, it is not known whether C-peptide passively diffuses across the cellular membrane or whether it is actively translocated by a specific pathway of internalisation, such as endocytosis. In this study, we investigated the hypothesis that C-peptide internalises in target cells by following a specific endocytic pathway.

The internalisation of C-peptide was explored in human aortic endothelial cells (HAEC) and umbilical artery smooth muscle cells (UASMC), two important targets of C-peptide activity, especially in the context of vascular dysfunction leading to vascular complications in type 1 diabetes [13-16]. We found that in these cells C-peptide internalises from the cellular surface within punctate structures, most of them co-localizing with early endosomes. A minor fraction of C-peptide vesicles, which increases with culture time, is localised within the lysosomes for degradation. These results demonstrate that C-peptide internalises in target cells by using a specific endocytic pathway. The endosome localisation of Cpeptide would support the proposal that C-peptide might achieve its cellular effects in part by signalling from these organelles.

\section{Methods}

Cell culture HAEC and UASMC were obtained from Cambrex Bioscience Walkersville (Walkersville, MD, USA) and grown in $75 \mathrm{~cm}^{2}$ flasks (Corning, Corning, NY, USA) at $37^{\circ} \mathrm{C}$ under $5 \% \mathrm{CO}_{2}$ in the presence of endothelial basal medium-2 supplemented with endothelial growth medium (HAEC) or smooth muscle cell basal medium-2 (SMGM-2; UASMC) (all from Cambrex Bioscience). To the SMGM- 2 was added $5 \%$ FCS, $0.1 \%$ antibiotics GA1000 (gentamicin, amphotericin B), 0.2\% human basic fibroblast growth factor (hFGF-b), $0.1 \%$ insulin, and $0.1 \%$ human epidermal growth factor (hEGF) (Cambrex Bioscience). HAEC were used at passage 2-6 and UASMC were used at passage 4-10. Unless differently specified, in all experiments HAEC and UASMC ( 100,000 cell/dish) were seeded on poly-D-lysine-coated MatTek plates (MatTek, Ashland, MA, USA) $35 \mathrm{~mm}$ in diameter, and allowed to settle overnight at $37^{\circ} \mathrm{C}, 5 \% \mathrm{CO}_{2}$ in their specific medium. Next day, cells were washed with fresh medium before starting with the specific experimental conditions.

Preparation of Alexa Fluor-labelled C-peptide and culture conditions We designed two Alexa Fluor-labelled Cpeptide probes, one labelled with Alexa Fluor 488 and the other with Alexa Fluor 546, and had them synthesised by Molecular Probes (Invitrogen, Carlsbad, CA, USA). Unless differently specified, $1 \mathrm{mmol} / \mathrm{l}$ of Alexa Fluor 488- or Alexa Fluor 546-labelled C-peptide (Molecular Probes) was added to each MatTek plate in serum-free medium. Cells were incubated at $37^{\circ} \mathrm{C}, 5 \% \mathrm{CO}_{2}$ for the designated time. After incubation, fluorescent probe was discarded and the cells were washed with fresh medium before analysis under a confocal microscope. The concentration of fluorescent probe corresponded to the minimum concentration of fluorescent probe that we were able to easily detect in our experimental system, and had also been previously used by others [12]. Details about probe synthesis and use in the experiments can be found in the Electronic supplementary material (ESM).

\section{Live-cell confocal imaging of C-peptide internalisation}

Cells were incubated with the fluorescent $\mathrm{C}$-peptide probe for a minimum of $5 \mathrm{~min}$ and a maximum of $1 \mathrm{~h}$. As a control for specificity of staining, $1 \mathrm{mmol} / \mathrm{l}$ of free Alexa Fluor 488 (catalogue no. A10235; Molecular Probes) or Alexa Fluor 546 fluorescent dye (catalogue no. A20002; Molecular Probes) was added to separate plates. Cells were studied in an 'open' cell cultivation system at $37^{\circ} \mathrm{C}$ by livecell confocal microscopy 5, 10, 20,30 min and $1 \mathrm{~h}$ after uptake of the C-peptide probe. At least eight independent experiments were performed, each with a minimum of six MatTek plates with adherent cells. 
Effect of temperature on Alexa Fluor-labelled C-peptide internalisation Cells were cooled at $4^{\circ} \mathrm{C}$ in a refrigerator for $1 \mathrm{~h}$ and then incubated with the fluorescent $\mathrm{C}$-peptide probe at $4^{\circ} \mathrm{C}$ for $30 \mathrm{~min}$. Uptake of the fluorescent probe by live cells was studied under a confocal microscope. Cells were then incubated at $37^{\circ} \mathrm{C}$ under $5 \% \mathrm{CO}_{2}$ for $1 \mathrm{~h}$ and analysed again with a confocal microscope. At least four independent experiments were performed, each with a minimum of six MatTek plates.

Inhibition of Alexa Fluor-labelled C-peptide uptake by excess of unlabelled $C$-peptide Cells were incubated with either $30 \mathrm{mmol} / 1$ of unlabelled native C-peptide (Sigma Chemical, St Louis, MO, USA) or $30 \mathrm{mmol} / 1$ of scrambled C-peptide (Sigma-Genosys, The Woodlands, TX, USA) for $1 \mathrm{~h}$ at $37^{\circ} \mathrm{C}, 5 \% \mathrm{CO}_{2}$. The latter peptide (purity $>95 \%$ ) is a randomised version of the native $\mathrm{C}$-peptide containing the same amino acid residues but randomly ordered, as follows: ADQEVELGAPQSAGLGGSQLEPQGLGLVLGE. After $1 \mathrm{~h}$ of incubation, fluorescent C-peptide probe was added for $30 \mathrm{~min}$ at $37^{\circ} \mathrm{C}, 5 \% \mathrm{CO}_{2}$. Live cells were then analysed under a confocal microscope. Uptake of C-peptide in these cells was compared with uptake of fluorescent $\mathrm{C}$-peptide in the absence of any preincubation with unlabelled, native or scrambled C-peptide. Three independent experiments were performed, each with a minimum of three MatTek plates.

Uptake of Alexa Fluor-labelled C-peptide in the presence of pharmacological compounds Cells were pretreated for $30 \mathrm{~min}$ at $37^{\circ} \mathrm{C}$, under $5 \% \mathrm{CO}_{2}$ with either monodansylcadaverine (MDC; $43 \mathrm{mmol} / \mathrm{l}$ ), which inhibits the clathrincoated pit pathway, filipin $(5 \mathrm{mg} / \mathrm{ml})$, which blocks the caveola pathway, nocodazole $(10 \mathrm{mmol} / \mathrm{l})$, which disrupts microtubule assembly, or cytochalasin D (30 $\mathrm{mmol} / \mathrm{l})$, which disrupts actin filaments. The dose and duration of treatment for each compound, none of which showed any toxic effect, was determined experimentally on the basis of previous reports [17-20]. After $30 \mathrm{~min}$, fluorescent Cpeptide was added for $30 \mathrm{~min}$ and the cells were put back in the incubator. Cells were then examined under a confocal microscope. Three independent experiments were performed, each with a minimum of three MatTek plates. The response of cells to each condition was highly consistent with inhibition of C-peptide uptake with MDC and nocodazole. We therefore analysed the mean fluorescence per cell in only three cells per plate (total of nine cells) for each condition, using Fluoview 300 software (Olympus, Melville, NY, USA). Repeated measures ANOVA was used to determine statistical significance.

Live-cell confocal imaging of C-peptide co-localisation with subcellular organelles Cells were transduced with $500 \mathrm{ml}$ Organelle Lights Endosomes-GFP reagent (cata- logue no. 010104; Invitrogen), which targets RAB5A, an early endosomal marker, following the manufacturer's instructions. The plates were incubated for $2 \mathrm{~h}$ on a plate shaker at room temperature. After removing the medium, $500 \mathrm{ml}$ of 1:1,000 dilution of enhancer was added to the cells and the plates were incubated at $37^{\circ} \mathrm{C}$ under $5 \% \mathrm{CO}_{2}$ for $2 \mathrm{~h}$. Cells were kept in the incubator for 2 days in fresh medium to allow production of the fluorescence protein, then incubated with fluorescent C-peptide for $30 \mathrm{~min}$ before examination by confocal microscopy. The same method was used to study the localisation of C-peptide in lysosomes and mitochondria; details are presented in the ESM. At least five independent experiments were performed for each organelle, each with a minimum of six MatTek plates with adherent cells.

Localisation of C-peptide in early endosomes by immunohistochemistry HAEC ( 100,000 cell/dish) seeded on MatTek plates were fixed with paraformaldehyde and incubated with rabbit anti-human antibody to C-peptide (Millipore, Billerica, MA, USA) together with a mouse anti-human monoclonal antibody to the early endosomal antigen 1 (EEA1) (Santa Cruz Biotechnology, Santa Cruz, CA, USA) for $2 \mathrm{~h}$. Appropriate secondary antibodies were then applied. Cells were washed and imaged using confocal microscopy. For a detailed description of the method, see the ESM. At least four independent experiments were performed, each with a minimum of six MatTek plates with adherent cells.

Isolation of endosomes and analysis of C-peptide content To identify early endosomes, we followed the internalisation of Alexa Fluor 488-conjugated human transferrin, a ligand known to enter the cells by clathrin-mediated endocytosis and a specific marker of early endosomes [21], in the presence of Alexa Fluor 546labelled C-peptide in UASMC in the incubator for $10 \mathrm{~min}$. Endosomes were isolated from other subcellular compartments by centrifugation (modified from [22]) and an aliquot of both the pellet and the endosome-containing supernatant fraction was used to quantitate Alexa Fluor 488-transferrin and Alexa Fluor 546-C-peptide fluorescence spectra by using a scanning spectrofluorometer. For a detailed description of the method and data analysis, see the ESM. Repeated measures ANOVA was used to determine statistical significance. Three independent experiments were performed.

Confocal fluorescence microscopy imaging Confocal fluorescence microscopy was performed using a Fluoview 300 Confocal Laser Scanning head (Olympus) with an IX70 inverted microscope (Olympus). Details of the procedure of cell imaging are presented in the ESM. 


\section{Results}

C-peptide internalises in human vascular endothelial and smooth muscle cells By using live-cell confocal laserscanning microscopy, we found that C-peptide bound to plasma membranes and internalised in the cytoplasm of HAEC (Fig. 1a) and UASMC (Fig. 1b). The uptake of Alexa Fluor 488-labelled C-peptide was minimal after $5 \mathrm{~min}$ and began to be clearly visible after $10 \mathrm{~min}$ of incubation. C-peptide uptake proceeded for the next $20 \mathrm{~min}$ and after $30 \mathrm{~min}$ of incubation fluorescent C-peptide was distributed along the plasma membrane and in the cytosol as punctate structures (Fig. 1a, b). Uptake was complete in all cells of a plate within $1 \mathrm{~h}$ of incubation. HAEC and UASMC showed a similar timing of C-peptide uptake. Incubation of cells with Alexa Fluor 488 fluorescent dye alone resulted in the absence of staining (Fig. 1c).

Effect of temperature on internalisation of Alexa Fluorlabelled C-peptide Consistent with the cellular trafficking of proteins in general, the internalisation of C-peptide was blocked at $4^{\circ} \mathrm{C}$ (Fig. 2a). Accordingly, C-peptide internalisation slowly recovered when cells that were incubated at $4^{\circ} \mathrm{C}$ were placed back at $37^{\circ} \mathrm{C}$ (Fig. $2 \mathrm{~b}, \mathrm{c}$ ).

Uptake of Alexa Fluor-labelled C-peptide is blocked by preincubation with unlabelled C-peptide As shown in Fig. 3, preincubation of HAEC with a 30 -fold excess of unlabelled C-peptide blocked uptake of the fluorescent Cpeptide probe compared with cells incubated with Alexa Fluor 488-labelled C-peptide only (Fig. 3a-c). On the contrary, preincubation of HAEC with a scrambled version of unlabelled C-peptide did not interfere with Alexa Fluor 488-labelled C-peptide internalisation (Fig. 3d).

C-peptide uses a clathrin-mediated endocytotic pathway to enter HAEC We investigated whether internalisation of C- peptide was mediated through clathrin-coated pits or caveolae by using MDC and filipin. MDC is a pharmacological inhibitor of receptor-mediated endocytosis [18, 21], while filipin, which binds cholesterol in the plasma membrane, impairs the invagination and subsequent internalisation of caveolae [23, 24]. Under our experimental conditions, pretreatment of HAEC with MDC (43 mmol/l) for 30 min completely blocked the uptake of Alexa Fluor 488-labelled C-peptide ( $p<0.01$ vs control) (Fig. 4). By contrast, pretreatment of HAEC with filipin for $30 \mathrm{~min}$ $(5 \mathrm{mg} / \mathrm{ml})$ had almost no inhibitory effect on fluorescent Cpeptide probe internalisation (Fig. 4).

Role of the cytoskeleton network The involvement of the cytoskeleton network in C-peptide endocytosis was studied in HAEC with cytochalasin D $(30 \mathrm{mmol} / \mathrm{l})$ and nocodazole $(10 \mathrm{mmol} / \mathrm{l})$, which act by inducing depolymerisation of actin filaments and microtubules, respectively $[25,26]$. As shown in Fig. 4, uptake of Alexa Fluor 488-labelled Cpeptide after cytochalasin D treatment was not significantly different from that in control cells. Conversely, nocodazole drastically reduced fluorescent C-peptide uptake $(p<0.01$ vs control), suggesting that entry of C-peptide into HAEC depended on microtubule integrity.

C-peptide upon internalisation co-localises with early endosomes To explore the possibility that C-peptide localises in early endosomes, HAEC and UASMC were transduced with Organelle Lights Endosome-GFP reagent, which targets the production of fluorescent RAB5A, an early endosome-specific marker. Live cells were then labelled with $1 \mathrm{mmol} / 1$ Alexa Fluor 546-labelled Cpeptide and imaged under a confocal microscope. As shown in Fig. 5a, early endosomes appeared as green punctate structures in the cytoplasm, close to the cellular membrane of an endothelial cell. Figure $5 \mathrm{~b}$ shows the internalisation of Alexa Fluor 546-labelled C-peptide as
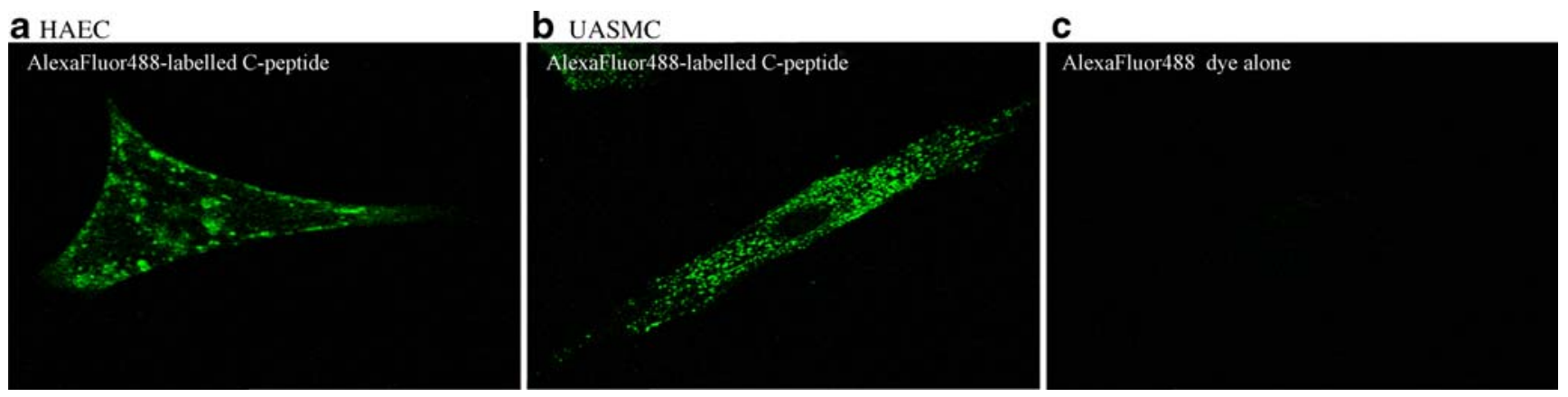

Fig. 1 C-peptide internalises in HAEC and UASMC as punctate structures. HAEC (a) and UASMC (b) were incubated for $30 \mathrm{~min}$ with $1 \mathrm{mmol} / 1$ Alexa Fluor 488 -labelled C-peptide at $37^{\circ} \mathrm{C}$, washed with medium and imaged by confocal microscopy. Green punctate staining corresponds to the C-peptide probe localised at the periphery of the cell and in the cytoplasm. c As a control for staining specificity, HAEC were incubated for $30 \mathrm{~min}$ with $1 \mathrm{mmol} / 1$ Alexa Fluor 488 dye at $37^{\circ} \mathrm{C}$. Note the absence of green fluorescent staining. The figure shows a representative z-section across one cell 


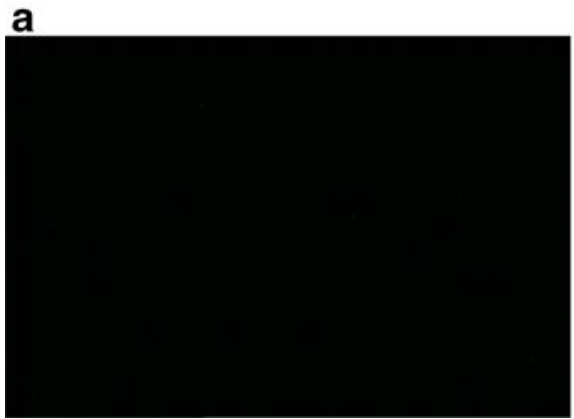

b

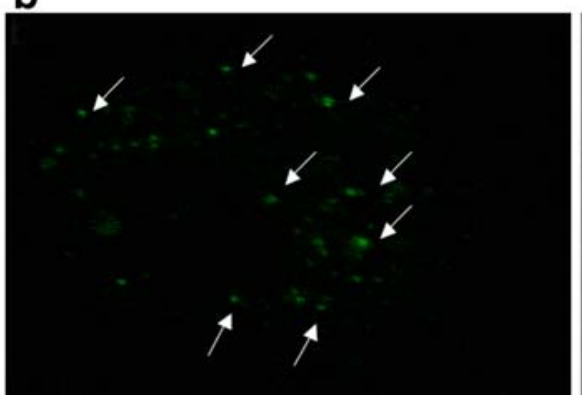

C

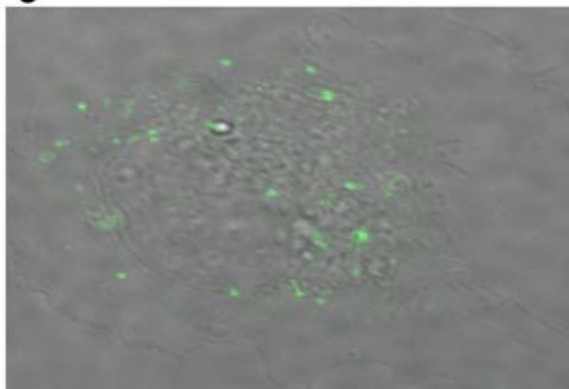

Fig. 2 C-peptide internalisation is inhibited at $4^{\circ} \mathrm{C}$. a HAEC were incubated for $30 \mathrm{~min}$ with $1 \mathrm{mmol} / 1$ Alexa Fluor 488-labelled Cpeptide at $4{ }^{\circ} \mathrm{C}$, washed with medium, and immediately imaged by confocal microscopy. The figure shows absence of green fluorescence staining inside the cells, consistent with no internalisation of the probe. b Internalisation of green C-peptide probe slowly recovered when cells were put in the incubator at $37^{\circ} \mathrm{C}$ after being at $4{ }^{\circ} \mathrm{C}$.
Arrows show examples of green punctate staining corresponding to the internalised C-peptide probe. c Differential interference contrast confocal image of the same z-section as in b merged with the fluorescence image to demonstrate localisation of green punctate staining in the cytoplasm. The figure shows a representative z-section across one endothelial cell a

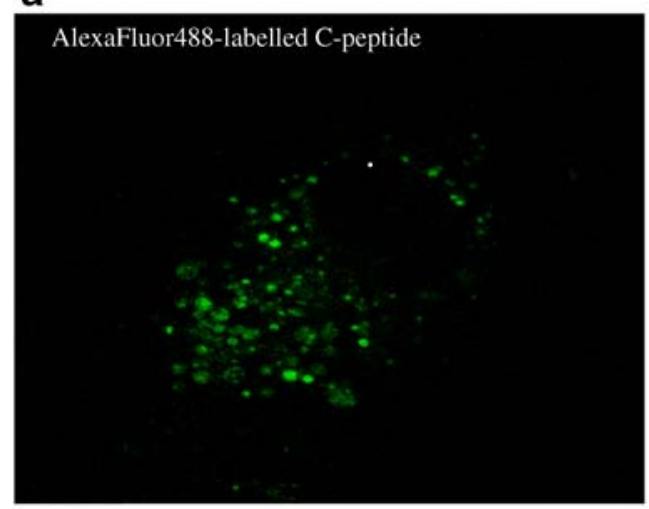

C

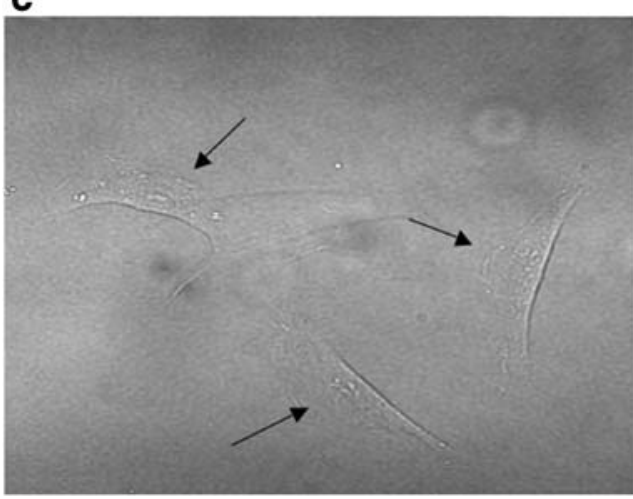

Fig. 3 Alexa Fluor 488-labelled C-peptide internalisation is inhibited by an excess of unlabelled C-peptide. a HAEC were incubated for $30 \mathrm{~min}$ with $1 \mathrm{mmol} / 1$ Alexa Fluor 488 -labelled C-peptide at $37^{\circ} \mathrm{C}$, washed with medium, and immediately imaged by confocal microscopy. The figure shows green punctate staining inside the periphery and cytoplasm of the cell, consistent with internalisation of the probe. b Internalisation of the Alexa Fluor 488-labelled green C-peptide probe $(1 \mathrm{mmol} / \mathrm{l})$ was inhibited by preincubation with $30 \mathrm{mmol} /$ 1 unlabelled C-peptide for $1 \mathrm{~h}$ at $37^{\circ} \mathrm{C}$. c Differential interference b

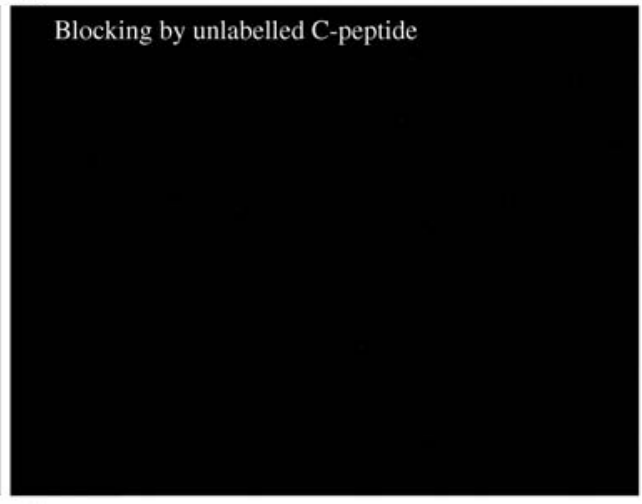

d

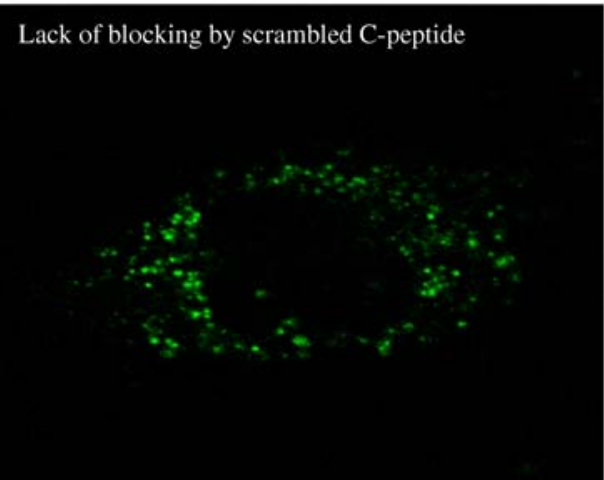

contrast confocal image of the z-section shown in b. Arrows indicate examples of three different cells showing reduced green punctate staining in the cytoplasm. d Preincubation of cells with $30 \mathrm{mmol} / \mathrm{l}$ of a randomised version of C-peptide (scrambled C-peptide) did not affect internalisation of Alexa Fluor 488-labelled C-peptide, as demonstrated by the presence of green punctate staining inside the cytoplasm. The figure shows a representative z-section across one or few endothelial cells 

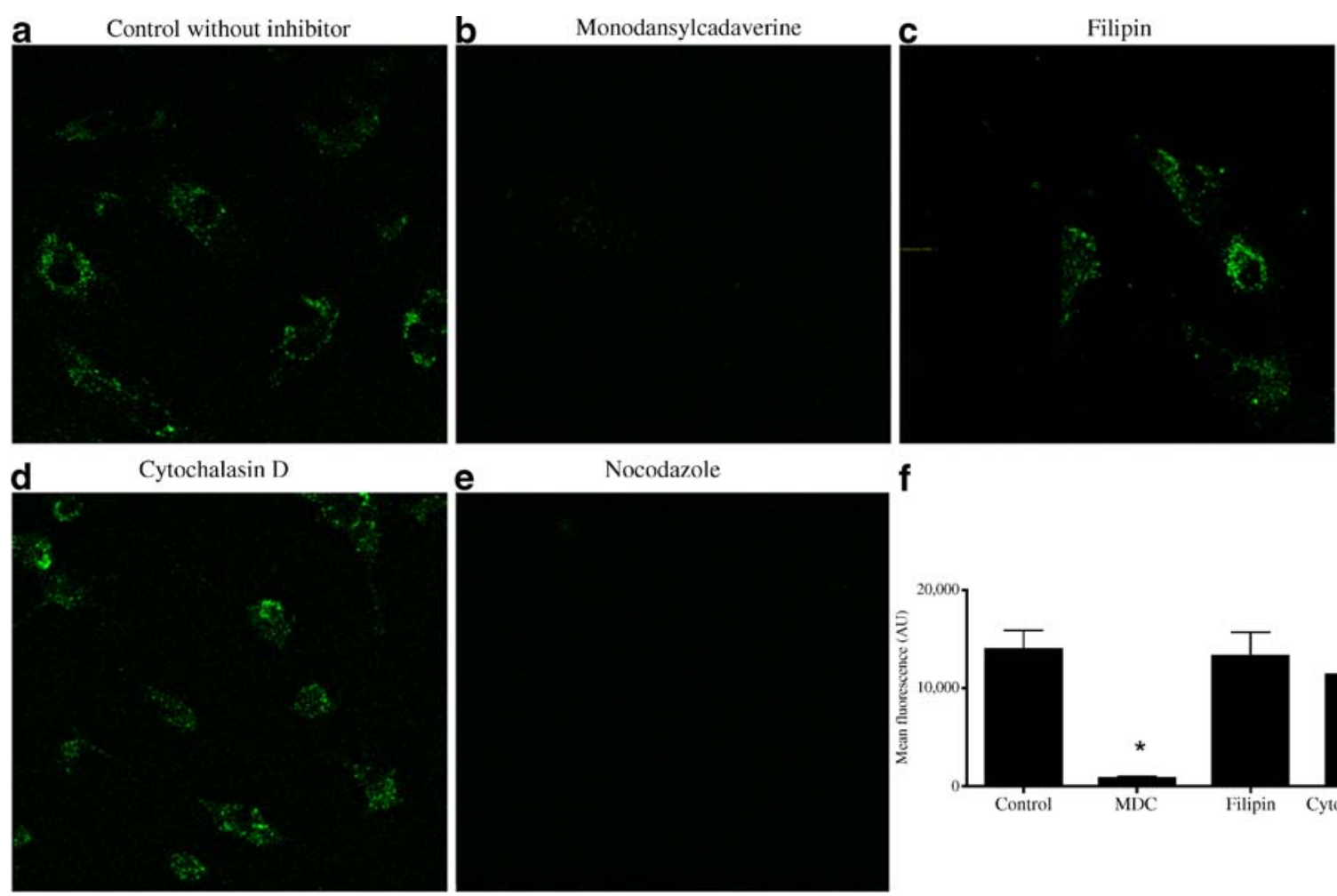

f

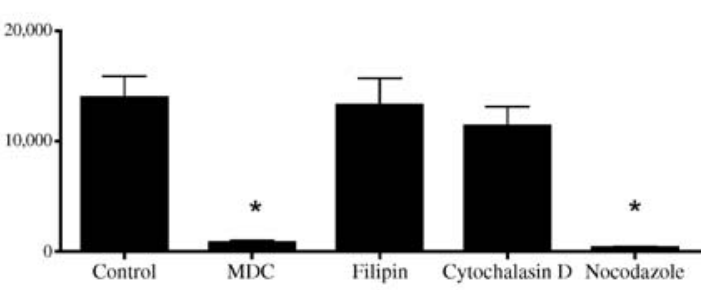

Fig. 4 Effect of different pharmacological compounds on C-peptide entry into HAEC. Cells were either treated with $1 \mathrm{mmol} / 1$ Alexa Fluor 488-labelled C-peptide for $30 \mathrm{~min}$ (control) or pretreated with the following compounds before addition of $1 \mathrm{mmol} / \mathrm{l}$ Alexa Fluor 488labelled C-peptide: MDC (43 mmol/l), filipin $(5 \mathrm{mg} / \mathrm{ml})$, cytochalasin $\mathrm{D}(30 \mathrm{mmol} / \mathrm{l})$ and nocodazole $(10 \mathrm{mmol} / \mathrm{l})$. As shown, pretreatment of HAEC with MDC, an inhibitor of clathrin-mediated endocytosis, inhibited entry of the C-peptide probe. Pretreatment with nocodazole,

red punctate staining within the cytosol. When the two images were merged, yellow staining was evident, corresponding to co-localisation of the green early endosomes with the red C-peptide (arrows in Fig. 5c). In most cases the red staining corresponding to the internalised C-peptide probe was evidently contained inside the endosome structures, observed as bright yellow peripheral staining around the central red staining of the vesicles (Fig. 5c, d).

Similarly, in UASMC, internalised Alexa Fluor 546labelled C-peptide (red) (Fig. 6c) co-localised with early endosomes (green) (Fig. 6b), observed as yellow staining on merging the two images (Fig. 6a, d).

Immunohistochemical localisation of C-peptide to early endosomes As an additional demonstration of the colocalisation of C-peptide to endocytic organelles, we performed immunofluorescence studies on paraformaldehyde-fixed HAEC after exposure to $10 \mathrm{nmol} / \mathrm{l} \mathrm{C}$-peptide. As shown in Fig. 7a, C-peptide internalised in HAEC as green punctate structures, most of which localised with the red early endosomes (Fig. 7b) identified with a monoclonal antibody which prevents microtubule assembly, also impairs C-peptide internalisation. Filipin and cytochalasin D did not inhibit C-peptide internalisation. a-e Representative z-section across some endothelial cells. $\mathbf{f}$ The mean fluorescence (AU, arbitrary units) of Alexa Fluor 488conjugated C-peptide internalisation measured in each experimental condition. The asterisk indicates that a significant decrease in uptake of fluorescent C-peptide was detected when HAEC were pretreated with MDC and nocodazole $(p<0.01$ vs control cells)

to the early endosome antigen 1 (EEA1), resulting in yellow staining (Fig. 7c).

Uptake of Alexa Fluor-labelled C-peptide follows transferrin internalisation in UASMC To isolate early endosomes, we briefly incubated UASMC with Alexa Fluor 546-labelled Cpeptide together with Alexa Fluor 488-conjugated human transferrin, and then proceeded to cellular fractionation. Highspeed centrifugation allows plasma membranes and other cellular compartments to sediment in the pellet, while clathrincoated pits and early endosomes, which are relatively small molecules (100-200 nm) [22], remain in the supernatant. By using a scanning fluorometer, we quantified the fluorescence present in the endosome-containing supernatant fraction of the homogenised cells. As shown in Fig. 8, the mean fraction of the Alexa Fluor 488-transferrin fluorescence (peak emission at 516), in the endosome-containing supernatant fraction was $0.86 \pm 0.06(n=3)$. The mean fraction of Alexa Fluor 546-C-peptide (peak emission at $568 \mathrm{~nm}$ ) in the endosome-containing supernatant fraction was $0.77 \pm 0.08$ $(n=3)$. The fraction of each probe in the supernatant fraction vs pellet was significantly different $(p<0.001)$, whereas the 
Fig. 5 Internalised Alexa Fluor 546-labelled C-peptide

co-localises with early endosomes in HAEC. HAEC were

transduced with Organelle Lights Endosomes-GFP, which induces production of the fluorescent early endosomal marker RAB5A. Cells were then incubated for $30 \mathrm{~min}$ with $1 \mathrm{mmol} / \mathrm{l}$ Alexa Fluor 546-labelled C-peptide at $37^{\circ} \mathrm{C}$, washed with medium and imaged by confocal microscopy in an ‘open' live-cell setting. a Green RAB5A staining in early endosomes. b Internalisation of Alexa Fluor 546-labelled

C-peptide visible as red vesicles in the cytosol. c Co-localisation of C-peptide (red) with early endosomes (green) appears as yellow staining. d Enlarged image of co-localisation shown as yellow staining. Arrows point to examples of co-localisation. The panels show a representative $\mathrm{z}$-section across one endothelial cell

Fig. 6 Internalised Alexa Fluor 546-labelled C-peptide colocalised with early endosomes in UASMC. UASMC were transduced with Organelle Lights Endosomes-GFP, which induces production of the fluorescent early endosomal marker RAB5A, and then incubated for 30 min with $1 \mathrm{mmol} / 1$ Alexa Fluor 546-labelled C-peptide at $37^{\circ} \mathrm{C}$. After washing the cells with fresh medium, cells were imaged by confocal microscopy. a Yellow staining corresponds to merging of green fluorescence (early endosomes) with red fluorescence (C-peptide). b-d Images of part of the cell within the red quadrant. Alexa Fluor 546-labelled C-peptide colocalised, or was close to, early endosomes (arrows). The panels show one representative z-section across one smooth muscle cell a

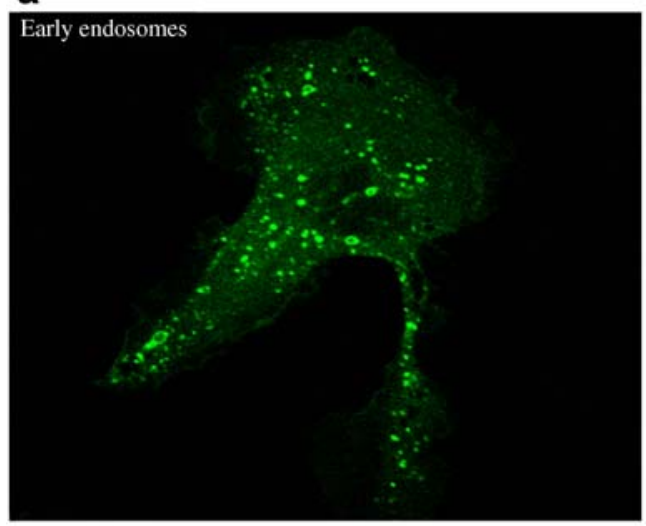

C

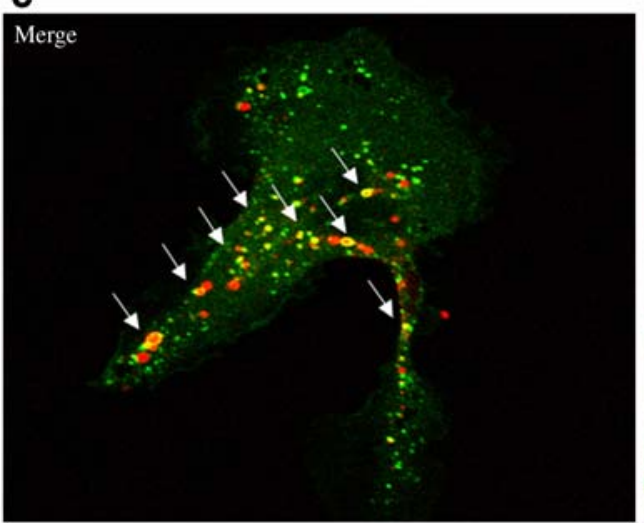

a UASMC

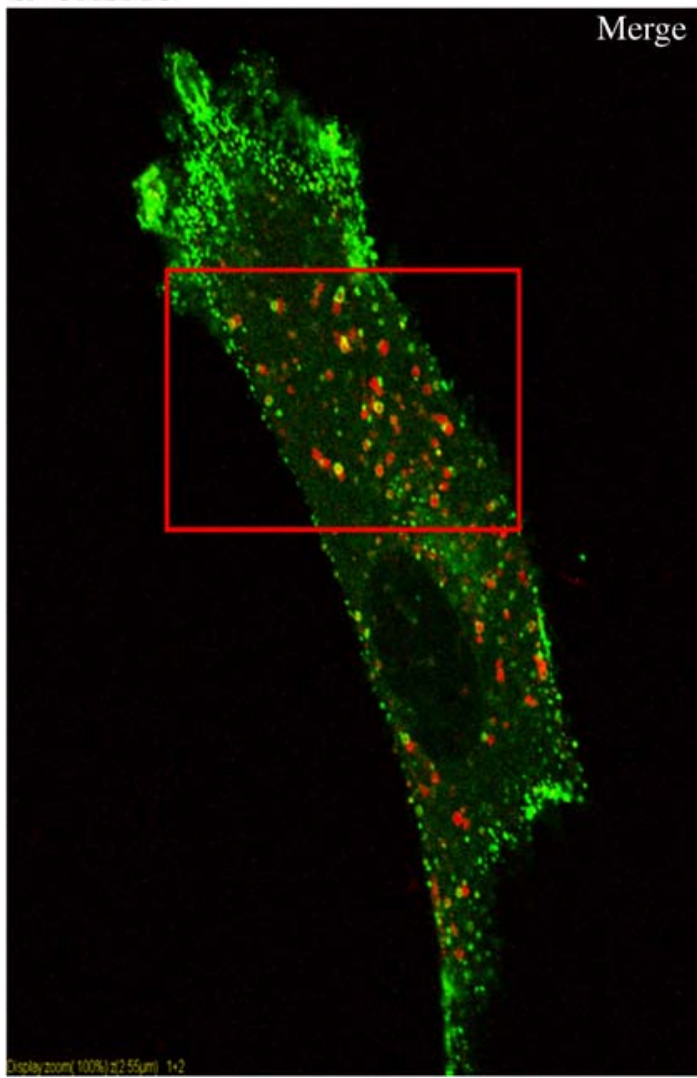

\section{d}

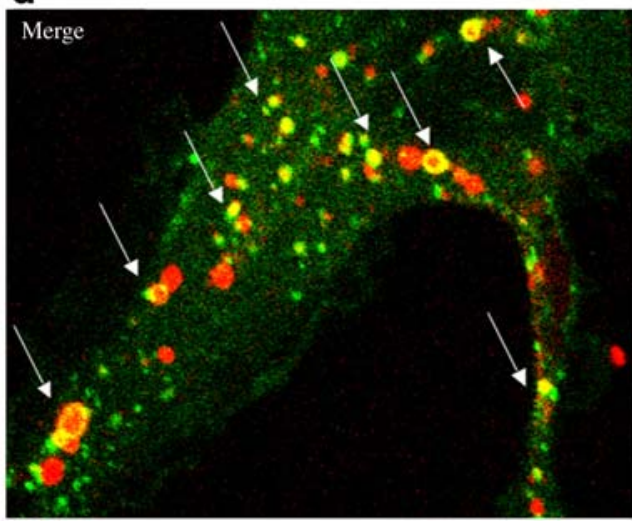

b
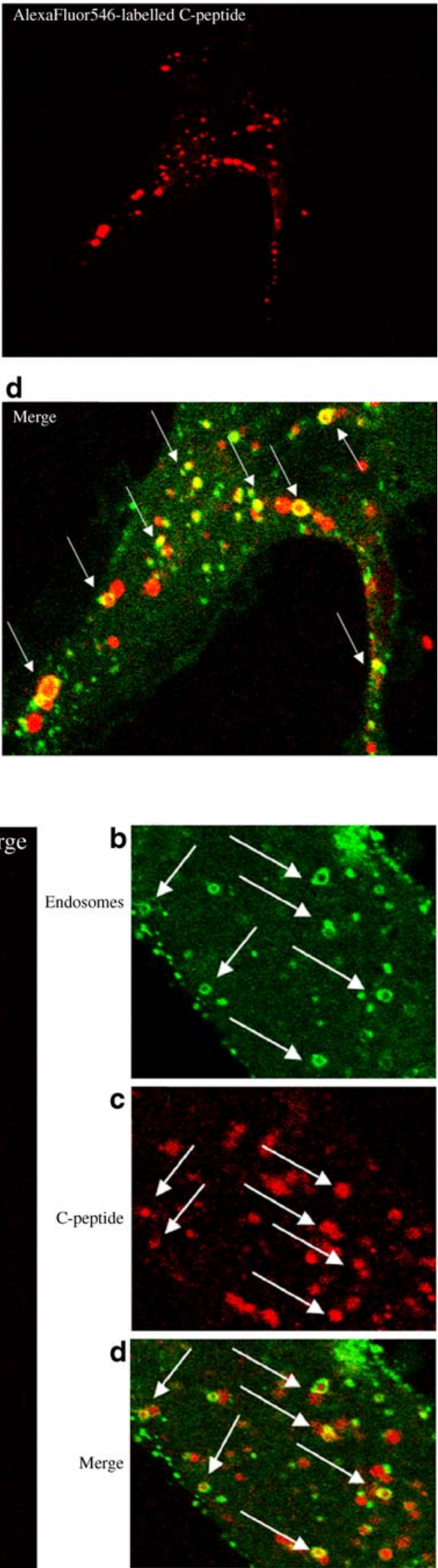

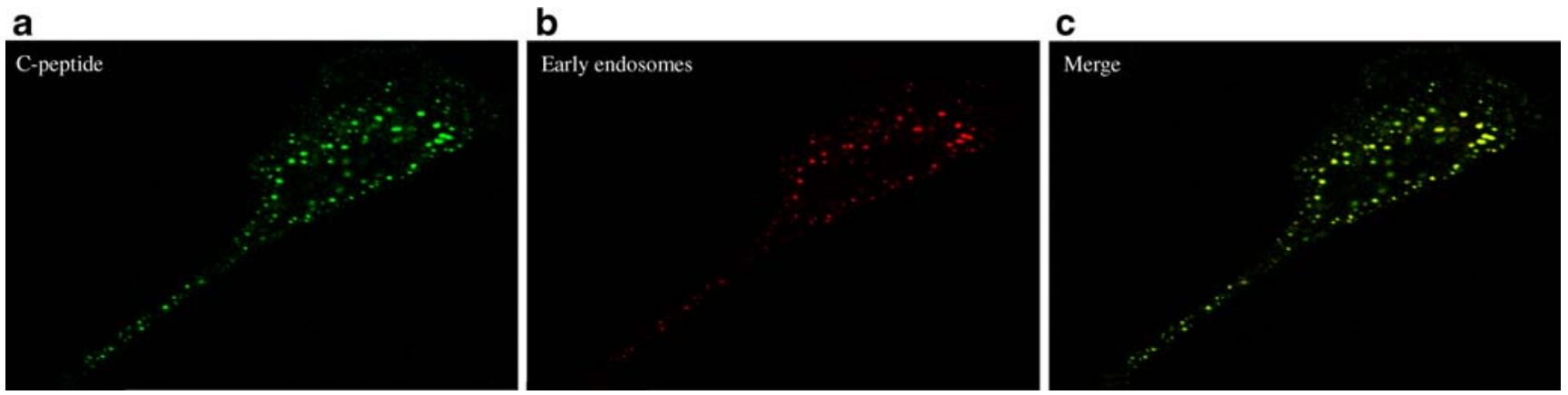

Fig. 7 Immunohistochemistry of C-peptide internalisation and localisation to early endosomes. HAEC were incubated for $3 \mathrm{~h}$ with $10 \mathrm{nmol} /$ $1 \mathrm{C}$-peptide at $37^{\circ} \mathrm{C}$. After fixation with paraformaldehyde, cells were incubated with rabbit anti-human antibody to C-peptide together with a mouse monoclonal antibody to the early endosome antigen 1 (EEA1) for

fractions of transferrin and C-peptide in the endosomal fraction were statistically indistinguishable $(p>0.05)$. The cell fractionation results further support the internalisation of C-peptide into early endosomes.

C-peptide traffics to lysosomes for degradation The final destination of internalised peptides along the endocytic pathway includes the lysosomes, where proteolytic degradation occurs. To visualise lysosomes, cells were transduced with Organelle Lights Lysosome-RFP reagent, which targets the lysosome-specific protein lysosomal-associated membrane protein 1 (LAMP1), before labelling the cells with fluorescent C-peptide. By live-cell confocal microscopy, we found that a fraction of green Alexa Fluor 488-labelled Cpeptide vesicles (Fig. 9a) co-localised with the red lysosomes (Fig. 9b), observed as yellow staining (Fig. 9c). The fraction

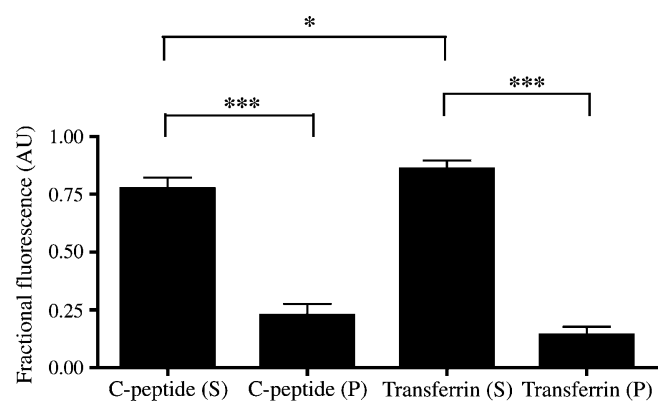

Fig. 8 C-peptide co-localises with isolated early endosomes in UASMC. To identify early endosomes, we studied the internalisation of Alexa Fluor 488-conjugated human transferrin, a specific marker of early endosomes, in the presence of Alexa Fluor 546-labelled Cpeptide in UASMC at $37^{\circ} \mathrm{C}, 5 \% \mathrm{CO}_{2}$ for $10 \mathrm{~min}$. Endosomes were isolated from other subcellular compartments by high-speed centrifugation and an aliquot of both the pellet $(\mathrm{P})$ and of the endosomecontaining supernatant fraction (S) was used to quantitate the Alexa Fluor 488-transferrin and Alexa Fluor 546-C-peptide fluorescence spectra by using a scanning spectrofluorimeter. The fluorescence of each molecule was expressed as a fraction of the total for each probe. AU, arbitrary units. ${ }^{*} p>0.05, * * * p<0.001$
$2 \mathrm{~h}$ and then with the appropriate secondary antibodies (see Methods). Cells were then washed and imaged by confocal microscopy. a Internalised C-peptide seen as green fluorescence vesicles. b Early endosomes, stained red. c Merging the two images shows colocalisation of most of the $\mathrm{C}$-peptide with endosomes

of C-peptide that co-localised with lysosomes increased with time in culture.

C-peptide does not appear to localise in mitochondria We did not find evidence of localisation of fluorescent Cpeptide in mitochondria of live HAEC and UASMC (data not shown).

\section{Discussion}

C-peptide is the peptide segment connecting the insulin A and $\mathrm{B}$ chains, and a product of proinsulin cleavage in the secretory granules, generated in pancreatic beta cells as part of normal insulin production. For a long time the only biological activity of C-peptide was considered to be to favour proinsulin folding within beta cells. Recent studies have, however, challenged this classical view by demonstrating that C-peptide is capable of biological effects in many different cell types and, importantly, reduces the complications associated with type 1 diabetes [2, 5-8]. Previous work from our laboratory has demonstrated a protective effect of C-peptide on the vasculature in hyperglycaemic conditions by decreasing inflammatory damage and the activation of nuclear factor (NF)- $\mathrm{KB}[13,15]$. More recently, a physiological role in glucose clearance and the release of ATP from erythrocytes of diabetic patients has been suggested [27]. C-peptide has also been shown to improve erythrocyte deformability in diabetic people [28].

The cell biology of C-peptide is for the most part unknown, in particular regarding the pathway the peptide uses to cross the cellular membrane and possible subcellular destinations. This study investigated the trafficking pathway of C-peptide in target cells upon its binding to cellular membranes. To this purpose, we designed Alexa Fluor-labelled C-peptide probes and followed their uptake in HAEC and UASMC, two 

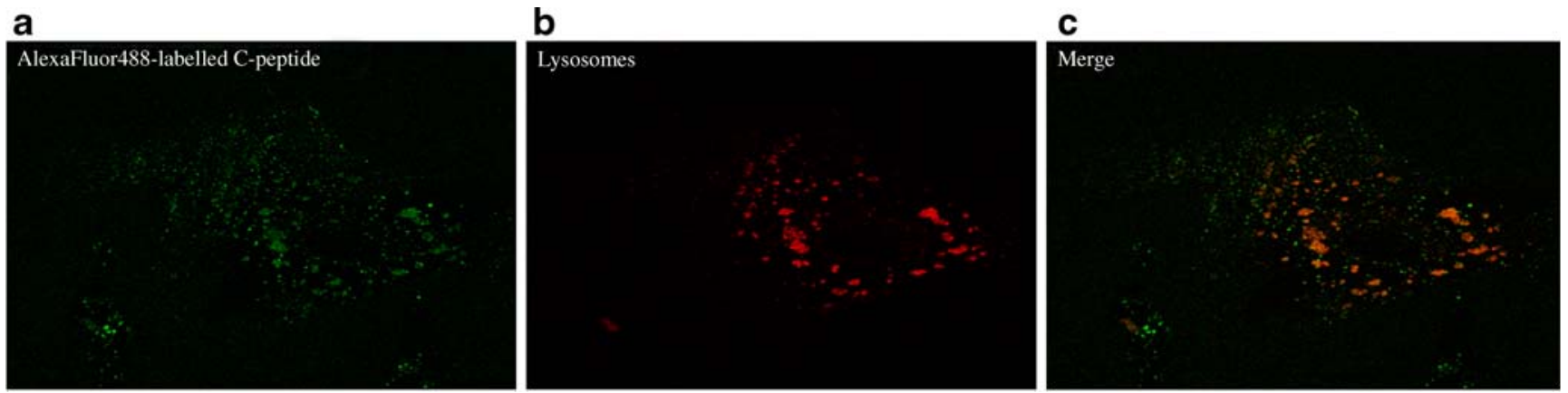

Fig. 9 Internalised Alexa Fluor 488-labelled C-peptide traffics to lysosomes in UAMSC. UASMC were transduced with Organelle Lights Lysosomes-RFP, which induces production of fluorescent LAMP1 protein, a specific lysosomal marker. UASMC were then incubated for $30 \mathrm{~min}$ with $1 \mathrm{mmol} / 1$ Alexa Fluor 488-labelled C-

relevant targets of C-peptide activity, by employing confocal laser scanning microscopy. Compared with spectrally similar conjugates, the Alexa Fluor fluorescent dyes have brighter fluorescence and are more photostable, which allows more time for image capture. Our findings in HAEC and UASMC demonstrate that $\mathrm{C}$-peptide internalises to punctate structures localised at the level of the cellular membrane and in the cytoplasm of live cells. The process of internalisation was minimal 5 min after the addition of the peptide and became clearly detectable after $10 \mathrm{~min}$. C-peptide uptake proceeded for $20 \mathrm{~min}$, resulting in bright staining in the cytoplasm after $30 \mathrm{~min}$ of incubation at $37^{\circ} \mathrm{C}$. The process was followed for $1 \mathrm{~h}$, after which internalisation was complete in the entire cell population. The process was mediated by $\mathrm{C}$-peptide, as the addition of Alexa Fluor dyes alone did not result in any intracellular staining. Specificity of C-peptide binding was also demonstrated by preincubation of the cells with an excess of unlabelled C-peptide, which blocked the internalisation of the C-peptide probe. Lindahl et al. [12] observed similar kinetics of C-peptide internalisation in HEK-293 cells and Swiss 3 T3 fibroblasts using a rhodamine-labelled C-peptide probe. As an additional technique to study C-peptide internalisation, we performed immunohistochemistry on endothelial cells exposed to $10 \mathrm{nmol} / \mathrm{l}$ of C-peptide, a concentration close to the physiological levels of C-peptide detected in healthy individuals after a meal. With this technique also, we were able to clearly detect C-peptide internalised inside the cytoplasm and at the level of the plasma membrane of endothelial cells as punctate structures.

The internalisation of C-peptide from the plasma membrane to the cytoplasm raises important biological questions. The first question concerns the fate of C-peptide upon internalisation. Unique to this work was our finding that Cpeptide traffics from the cell surface to early endosomes. This was demonstrated by a striking co-localisation of the red punctate structures corresponding to the Alexa Fluor-labelled $\mathrm{C}$-peptide probe with the green fluorescent circular organelles peptide at $37^{\circ} \mathrm{C}$, washed with medium and imaged by confocal microscopy. Yellow staining in c corresponds to the merging of green fluorescence (a, C-peptide) with red fluorescence (b, lysosomes), indicative of localisation. The figure shows images of a representative z-section across one vascular smooth muscle cell

corresponding to the RAB5A staining of early endosomes in HAEC and UASMC. Immunohistochemistry studies on endothelial cells exposed to nanomolar concentrations of C-peptide also demonstrated co-localisation of internalised Cpeptide with another early endosomal marker, EEA1. As another approach to study C-peptide internalisation, we isolated early endosomes from other subcellular compartments by high-speed centrifugation. As a marker of early endosomes, we used an Alexa Fluor 488 conjugate of human transferrin, which rapidly enters the cells through clathrinmediated endocytosis and localises in early endosomes. We found that most of the Alexa Fluor 546-labelled C-peptide colocalised in transferrin-positive endosomes, as also shown by quantification of C-peptide specific fluorescence in the supernatant fraction. The fact that not all internalised Cpeptide co-localised with the transferrin-positive endosomes is probably due to the different kinetics of internalisation of the two substrates in UASMC, transferrin internalisation being faster. Overall, these findings indicate a process of Cpeptide internalisation from the cell surface within membranebound organelles of the endocytic pathway, and exclude direct translocation across the plasma membrane.

In support of an endocytic pathway, the uptake of Cpeptide in HAEC and UASMC was observed when cells were kept at $37^{\circ} \mathrm{C}$ but not at $4^{\circ} \mathrm{C}$, which is consistent with the internalisation of surface protein receptors in general, a finding also reported previously [12]. C-peptide internalisation evidently proceeds by clathrin-mediated endocytosis, as it was inhibited by MDC, a pharmacological compound used to inhibit receptor-mediated endocytosis from clathrincoated pits. Microtubule integrity appears to be important in facilitating the early stages of internalisation of C-peptide in HAEC, as pretreatment of cells with nocodazole, an inhibitor of microtubule assembly, impaired C-peptide endocytosis. Internalisation of $\mathrm{C}$-peptide in the low nanomolar range via endocytosis provides indirect evidence for the presence of a specific membrane receptor. Although a putative C-peptide 
receptor has not been identified, it has been suggested to be a G-protein-coupled receptor, as deduced from the effects of pertussis toxin $[11,12]$.

Another classical feature of endocytosis is represented by lysosomal degradation. We report here for the first time the presence of trafficking of C-peptide to lysosomes in HAEC and UASMC. This was demonstrated by colocalisation of the green C-peptide staining corresponding to the Alexa Fluor 488-labelled C-peptide probe with the specific lysosome marker Lamp-1 under a confocal microscope, giving rise to yellow fluorescence. Lysosome trafficking of C-peptide increased with culture time, compatible with trafficking of endocytosed material along the endocytic pathway from early endosomes to lysosomes.

Trafficking of C-peptide from the cell surface to early endosomes provides a possible platform for the intracellular signalling events initiated by C-peptide in target cells. Endosomes interact with a complex network of tubules and vesicles distributed throughout the cytoplasm, interconnected by a tightly controlled transport system [29]. In addition to their classical role as sorting stations for internalised activated receptor-peptide complexes on their way to lysosomal degradation, endosomes are emerging as crucial players in intracellular signalling [30]. Examples of signalling endosomes are those associated with epidermal growth factor receptors (EGFRs), whose downstream signalling factors, such as SHC-adaptor protein (SHC), growth factor receptor bound protein 2 (GRB2) and mammalian Son-of-sevenless (mSOS), were found not only on the plasma membrane but also on early endosomes [31], suggesting that EGFR signalling continues in this compartment [32]. Another example of signalling endosomes is the endosome associated with nerve growth factor, which was found to be bound to its activated receptor potassium transporter peripheral membrane component (TRKA) and phospholipase C-g1 in endocytic organelles [33].

Based on our findings, signalling from putative Cpeptide-receptor complexes might be initiated at the plasma membrane, continue from early endosomes, and terminate at lysosomes. The effect of C-peptide that we observed on the nuclear factor kappaB (NF-kB) pathway in both HAEC and UASMC $[13,15]$ might thus originate from C-peptidereceptor complex signalling from the endosomes, as has been demonstrated for certain Toll-like receptor pathways and other inflammatory pathways, which affect the activation of the NF-KB pathway from the endosomes [34, 35]. The mildly acidic $\mathrm{pH}$ of the sorting endosomes would then begin the dissociation of the C-peptide destined to lysosomes from its recycled receptor. The effect of Cpeptide on the NF- $\mathrm{KB}$ pathway might also originate, in part, from C-peptide signalling from the nucleus, as Lindahl et $a l$. [12] found nuclear localisation of rhodamine-labelled Cpeptide in Swiss fibroblasts and 3T3 cells. In this case, trafficking of C-peptide-receptor complexes from the plasma membrane to early endosomes might simply represent an intermediate station on the pathway to lysosomal degradation. Further experiments are required to determine the contributions of endosomal and nuclear Cpeptide signalling.

Localisation of C-peptide to early endosomes does not exclude the possibility of trafficking to other subcellular compartments. We tested the possibility of a mitochondrial localisation of C-peptide by using a specific fluorescent mitochondrial dye. We found little or no localisation of the Alexa Fluor 488-labelled C-peptide in mitochondria in HAEC and UASMC.

In conclusion, we demonstrate here for the first time that C-peptide, upon internalisation from the plasma membrane, co-localises with early endosomes in both HAEC and UASMC. This finding is suggestive of a process of internalisation via the classic endocytic pathway rather than by direct translocation across the plasma membrane. A minor fraction of internalised C-peptide is localised to lysosomes, while no apparent localisation of C-peptide was found in the mitochondria. Endosomes might represent a signalling station through which C-peptide might achieve its cellular effects, or reflect a transport intermediate on the way to lysosomal degradation.

Acknowledgements This study was supported by the Henry Hillman Endowment Chair in Pediatric Immunology (to M. Trucco) and by grants DK 024021-24 from the National Institute of Health, W81XWH-06-1-0317 from the Department of Defense (to M. Trucco), and 1-06-RA-39 from the American Diabetes Association (to P. Drain). We are grateful to L. M. Traub (Department of Cell Biology and Physiology, University of Pittsburgh, School of Medicine) for critical discussion about endosome isolation and G. Salama (Department of Cell Biology and Physiology, University of Pittsburgh, School of Medicine) for the use of the Jobin Yvon Fluolog 3 scanning spectrofluorometer.

Duality of interest statement The authors declare that there is no duality of interest associated with this manuscript.

\section{References}

1. Samnegard B, Brundin T (2001) Renal extraction of insulin and C-peptide in man before and after a glucose meal. Clin Physiol 21:164-171

2. Wahren J, Ekberg K, Jornvall H (2007) C-peptide is a bioactive peptide. Diabetologia 50:503-509

3. Hills CE, Brunskill NJ (2008) Intracellular signaling by C-peptide. Exp Diabetes Res. doi:10.1155/2008/635158

4. Vish M, Mangeshkar P, Piraino G et al (2007) Proinsulin C-peptide exerts beneficial effects in endotoxic shock in mice. Crit Care Med 35:1348-1355

5. Johansson BL, Borg K, Fernqvist-Forbes E, Kernell A, Odergren T, Wahren J (2000) Beneficial effects of C-peptide on incipient nephropathy and neuropathy in patients with type 1 diabetes: a three-month study. Diabet Med 17:181-189 
6. Ekberg K, Brismar T, Johansson BL et al (2007) C-peptide replacement therapy and sensory nerve function in type 1 diabetic neuropathy. Diabetes Care 30:71-76

7. Hansen A, Johansson BL, Wahren J, von Bibra H (2002) Cpeptide exerts beneficial effects on myocardial blood flow and function in patients with type 1 diabetes. Diabetes 51:3077-3082

8. Johansson BL, Wahren J, Pernow J (2003) C-peptide increases forearm blood flow in patients with type 1 diabetes via a nitric oxide-dependent mechanism. Am J Physiol Endocrinol Metab 285:E864-E870

9. Flatt PR, Swanston-Flatt SK, Hampton SM, Bailey CJ, Marks V (1986) Specific binding of the C-peptide of proinsulin to cultured B cells from a transplantable rat islet cell tumor. Biosci Rep 6:193-199

10. Pramanik A, Ekberg K, Zhong Z et al (2001) C-peptide binding to human cell membranes: importance of Glu27. Biochem Biophys Res Commun 284:94-98

11. Rigler R, Pramanik A, Jonasson P et al (1999) Specific binding of proinsulin C-peptide to human cell membranes. Proc Natl Acad Sci U S A 96:13318-13323

12. Lindahl E, Nyman U, Melles E et al (2007) Cellular internalization of proinsulin C-peptide. Cell Mol Life Sci 64:479-486

13. Luppi P, Cifarelli V, Tse H, Piganelli J, Trucco M (2008) Human C-peptide antagonizes high glucose-induced endothelial dysfunction through the NF-kB pathway. Diabetologia 51:1534-1543

14. Kobayashi Y, Naruse K, Hamada Y et al (2005) Human proinsulin $\mathrm{C}$ - peptide prevents proliferation of rat aortic smooth muscle cells cultured in high-glucose conditions. Diabetologia 48:2396-2401

15. Cifarelli V, Luppi P, Tse HM, He J, Piganelli J, Trucco M (2008) Human proinsulin C-peptide reduces high glucose-induced proliferation and NF-kappaB activation in vascular smooth muscle cells. Atherosclerosis 201:248-257

16. Marx N, Walcher D (2008) C-peptide and atherogenesis: Cpeptide as a mediator of lesion development in patients with type 2 diabetes mellitus. Exp Diabetes Res. doi:10.1155/2008/385108

17. Bradley JR, Johnson DR, Pober JS (1993) Four different classes of inhibitors of receptor-mediated endocytosis decrease tumor necrosis factor-induced gene expression in human endothelial cells. J Immunol 150:5544-5555

18. Schutze S, Machleidt T, Adam D et al (1999) Inhibition of receptor internalization by monodansylcadaverine selectively blocks p55 tumor necrosis factor receptor death domain signaling. J Biol Chem 274:10203-10212

19. Inal J, Miot S, Schifferli JA (2005) The complement inhibitor, CRIT, undergoes clathrin-dependent endocytosis. Exp Cell Res 310:54-65

20. Uriarte SM, Jog NR, Luerman GC, Bhimani S, Ward RA, McLeish KR (2009) Counter-regulation of clathrin-mediated endocytosis by the actin and microtubular cytoskeleton in human neutrophils. Am J Physiol Cell Physiol 296:C857-C867
21. Acosta EG, Castilla V, Damonte EB (2008) Functional entry of dengue virus into Aedes albopictus mosquito cells is dependent on clathrin-mediated endocytosis. J Gen Virol 89:474-484

22. Holroyd C, Kistner U, Annaert W, Jahn R (1999) Fusion of endosomes involved in synaptic vesicle recycling. Mol Biol Cell 10:3035-3044

23. Rothberg KG, Ying Y-S, Kamen BA, Anderson RG (1990) Cholesterol controls the clustering of the glycophospholipidanchored membrane receptor for 5-methyltetrahydrofolate. J Cell Biol 111:2931-2938

24. Schnitzer JE, Oh P, Pinney E, Allard J (1994) Filipin-sensitive caveolae-mediated transport in endothelium: reduced transcytosis, scavenger endocytosis, and capillary permeability of selected macromolecules. J Cell Biol 127:1217-1232

25. Flanagan MD, Lin S (1980) Cytochalasins block actin filament elongation by binding to high affinity sites associated with F-actin. J Biol Chem 255:835-838

26. Vasquez RJ, Howell B, Yvon AM, Wadsworth P, Cassimeris L (1997) Nanomolar concentrations of nocodazole alter microtubule dynamic instability in vivo and in vitro. Mol Biol Cell 8:973-985

27. Meyer JA, Froelich JM, Reid GE, Karunarathne WK, Spence DM (2008) Metal-activated C-peptide facilitate glucose clearance and the release of a nitric oxide stimulus via the GLUT1 transporter. Diabetologia 51:175-182

28. Hach T, Forst T, Kunt T, Ekberg K, Pfutzner A, Wahren J (2008) $\mathrm{C}$-peptide and its $\mathrm{C}$-terminal fragments improve erythrocyte deformability in type 1 diabetes patients. Exp Diabetes Res. doi: $10.1155 / 2008 / 730594$

29. Gruenberg J (2001) The endocytic pathway: a mosaic of domains. Nat Rev Mol Cell Biol 2:721-730

30. Miaczynska M, Pelkmans L, Zerial M (2004) Not just a sink: endosomes in control of signal transduction. Curr Opin Cell Biol $16: 400-406$

31. Di Guglielmo GM, Baass PC, Ou WJ, Posner BL, Bergeron JJ (1994) Compartmentalization of SHC, GRB2 and mSOS, and hyperphosphorylation of Raf-1 by EGF but not insulin in liver parenchyma. EMBO J 13:4269-4277

32. Baass PC, Di Guglielmo GM, Authier F, Posner BL, Bergeron JJ (1995) Compartmentalized signal transduction by receptor tyrosine kinases. Trends Cell Biol 5:465-470

33. Grimes ML, Zhou J, Beattie EC et al (1996) Endocytosis of activated trkA: evidence that nerve growth factor induces formation of signaling endosomes. J Neurosci 16:7950-7964

34. Husebye H, Halaas O, Stenmark H et al (2006) Endocytic pathways regulate Toll-like receptor 4 signaling and link innate and adaptive immunity. EMBO J 25:683-692

35. Dodeller F, Gotar M, Huesken D, Iourhenko V, Cenni B (2008) The lysosomal transmembrane protein 9B regulates the activity of inflammatory signaling pathways. J Biol Chem 283:21487-21494 